\title{
Editorial: The Rebound Effect and the Jevons' Paradox: Beyond the Conventional Wisdom
}

\author{
Franco Ruzzenenti ${ }^{*}$, David Font Vivanco ${ }^{2}$, Ray Galvin ${ }^{3}$, Steve Sorrell ${ }^{4}$, \\ Aleksandra Wagner ${ }^{5}$ and Hans Jakob Walnum ${ }^{6}$ \\ ${ }^{1}$ Center for Energy and Environmental Sciences, Faculty of Science and Engineering, University of Groningen, Groningen, \\ Netherlands, ${ }^{2}$ 2.-0 LCA Consultants, Aalborg, Denmark, ${ }^{3}$ Behaviour and Building Performance Group, Department of \\ Architecture, University of Cambridge, Cambridge, United Kingdom, ${ }^{4}$ Sussex Energy Group, SPRU-Science Policy Research \\ Unit, University of Sussex, Brighton, United Kingdom, ${ }^{5}$ Institute of Sociology, Jagiellonian University, Krakow, Poland, \\ ${ }^{6}$ Western Norway Research Institute, Sogndal, Norway
}

Keywords: rebound effect, Jevons' Paradox, complexity, moral licensing effect, circular economy, system-dynamics model, transdisciplanary paradigm

\section{Editorial on the Research Topic}

\section{The Rebound Effect and the Jevons' Paradox: Beyond the Conventional Wisdom}

The rebound effect (RE) is an umbrella term for a range of mechanisms that undermine the expected energy savings from improved energy efficiency. Since the seminal work of Stanley Jevons ("The Coal Question"), the "problem" of the rebound effect has repeatedly appeared in energy policy debates, challenging the consensus that improved energy efficiency will reduce energy use and carbon emissions and mitigate resource depletion. Most authors view energy efficiency as essential for reconciling economic growth with environmental sustainability, and consider rebound effects to be modest in size and easily addressed. However, there has always been a vocal minority who argue that rebound effects frequently exceed $100 \%$ and can potentially eliminate all of the energy savings from improved energy efficiency ("Jevons' Paradox"). This contentious and polarized debate has largely been confined to the academic world, with only occasional infringements into the public sphere. Until recently, the empirical research on rebound effects was also relatively modest, with $<10$ articles per year mentioning the topic between 1998 and 2008 .

However, academic interest in the topic has exploded since 2008, with more than 50 articles being published each year between 2015 and 2019. This suggests that that rebound effects are becoming increasingly recognized as a serious issue of concern and as an important field of investigation. The majority of these articles take the perspective of orthodox economics, and seek to estimate the size of rebound effects from historical data. However, as interest in the topic has grown, the diversity of perspectives, methodologies and approaches has increased, including contributions from a range of disciplines. This Research Topic of Frontiers in Energy Research and Frontiers in Sociology aims to capture some of these new perspectives, and prioritizes contributions that depart in significant ways from the economic orthodoxy.

The Research Topic consists of seven highly diverse contributions, which we briefly summarize below.

First, Santarius et al. discuss how disciplines such as psychology, sociology, and industrial ecology can contribute to the understanding of the rebound effect and highlight: first, the gap between the limits of any one analysis and the possible effects beyond those limits; and second, the challenge of isolating the causal influence of efficiency improvements from other relevant factors. They also discuss the implications of rebound effects for public policy. 
Arrobbio and Padovan analyse the dominant discourse on energy efficiency and suggest that the higher the expectations from energy efficiency, the lower the results in terms of reductions in energy use. They argue that these expectations may obstruct alternative strategies that are more effective in reducing environmental impacts.

Wallenborn provides a more in-depth discussion of the contribution of different disciplines to the understanding of rebound effects, and suggests ways in which their diverse insights can be synthesized. He argues that rebounds arise faster when infrastructures and markets enable energy to circulate, and when energy consumers are in competition.

Freeman develops a novel system-dynamics model of rebound effects which goes beyond traditional mechanisms to include economy-wide effects, transformational effects, frontier effects, and international rebound effects. Drawing upon the concepts of natural capital, ecological footprint and the great acceleration, she shows how such a model provides a useful "sand-box" for testing ideas about the future.

Dütschke et al. take a psychological perspective on rebound effects, and show how voluntary actions by individuals to reduce their environmental impacts can have unintended consequences. This is because people may feel they have "done their bit" for the environment and can subsequently spend time and money on more energy-intensive goods and activities-so-called "moral licensing." The authors develop a theoretical model that explains how economic and psychological motivations can trigger both rebound effects and moral licensing, and review the empirical evidence on the latter.

Makov and Font Vivanco go beyond the traditional focus on energy efficiency to show how rebound effects may apply to a circular economy strategies, such as reusing smartphones. They show how imperfect substitution between recycled and new products, together with re-spending of the cost savings, could erode around one third-and potentially all—of the emission savings from smartphone reuse.

Finally, Giampietro and Mayumi introduce a range of ideas from the theory of complex adaptive systems (e.g., holon, holarchy, Holling's adaptive cycle) to explain the systemic drivers of Jevons' Paradox. They argue that sustainability is based upon a dynamic balance of two contrasting thermodynamic principles-minimum entropy production and maximum energy flux-and show how this balance depends upon the biophysical constraints faced by the system. They argue that Jevons' Paradox is practically inevitable when biophysical constraints are not binding, but during phases in which society faces such constraints, efficiency improvements should be used to prevent the loss of existing diversity.

Taken together, the collection of articles demonstrate the limitations of the dominant, microeconomic framing of rebound effects and show how this phenomenon has much deeper roots in system behavior, human psychology, and social organization.
The articles also reinforce the argument that rebound effects are larger than many have assumed, and therefore present a critical challenge for environmental sustainability.

Until recently, the scientific debate on rebound effects has struggled to transfer into public discourse and to trigger political action. Organizations such as the International Energy Agency have largely ignored the phenomenon, and relatively few governments have taken specific measures to address rebound effects-partly because it remains unclear what measures are most appropriate. Perhaps the most widely recommended option is carbon pricing-either via carbon taxes or carbon emissions trading schemeswhich can offset the cost savings from improved energy efficiency and thereby dampen rebound effects. Carbon pricing remains politically challenging, however, and it is essential that the revenues are used in a way that minimizes the impact on low-income groups and increases public acceptance. It is also essential that regional and global energy scenarios make greater allowance for rebound effects, to avoid exaggerated expectations about the contribution of improved energy efficiency.

Perhaps it is also time for a broader public debate on this topic. Electronic media has significantly reduced the distance between academics and society and made the public space more accessible. Perhaps, scholars who are researching this fascinating subject should take some time to engage with people more broadly. But this is very challenging. Paradoxes, like the Jevons', are difficult to convey through a synthetic, captivating message and communication is often hindered by the specialization and fragmentation of contemporary knowledge. Hence, one of the challenges of social science is to present a holistic vision and to foster the imagination of possible, but not necessary intended or anticipated consequences. We hope this special edition can contribute to that end.

\section{AUTHOR CONTRIBUTIONS}

SS has written the text and all authors listed have made a substantial, direct and intellectual contribution to the work, and approved it for publication.

Conflict of Interest Statement: DF was employed by the company 2.-0 LCA consultants.

The remaining authors declare that the research was conducted in the absence of any commercial or financial relationships that could be construed as a potential conflict of interest.

Copyright (c) 2019 Ruzzenenti, Font Vivanco, Galvin, Sorrell, Wagner and Walnum. This is an open-access article distributed under the terms of the Creative Commons Attribution License (CC BY). The use, distribution or reproduction in other forums is permitted, provided the original author(s) and the copyright owner(s) are credited and that the original publication in this journal is cited, in accordance with accepted academic practice. No use, distribution or reproduction is permitted which does not comply with these terms. 